\title{
Ablation of SiN Passivation Layers on Photovoltaic Cells with Femtosecond Laser Source
}

\author{
K.Stolberg $^{* 1}$, S.Friedel ${ }^{* 1}$, B.Kremser ${ }^{* 1}$, M.Leitner $^{* 1}$, Y.Atsuta ${ }^{* 2}$ \\ *11 Jenoptik Laser, Optik, Systeme GmbH, Goeschwitzer Str. 25, 07745 Jena, Germany \\ klaus.stolberg@jenoptik.com \\ *22 Optopia Co.Ltd., KSP 714B, 3-2-1, Sakado, Takatsu-ku, Kawasaki-shi, Kanagawa, 213-0012 Japan \\ atsuta@optopia.co.jp
}

This paper presents results of high-speed selective laser ablation of Silicon Nitride (SiN) coatings, which are used as antireflection and passivation layer at monocrystalline and multicrystalline silicon wafers for solar cells. We discuss ablation mechanism of NIR femtosecond laser pulses and advantages for cold ablation with minimised lattice damage. We show practical results of singleshot ablation with a repetition rate of $100 \mathrm{kHz}$. Finally we compare layer ablation with NIR picosecond laser.

DOI: $10.2961 / \mathrm{j} 1 \mathrm{mn} .2010 .02 .0005$

Keywords: laser scribing, monocrystalline silicon, multicrystalline silicon, solar cell, selective emitter, antireflection layer, passivation layer, femtosecond laser, LAMP2009

\section{Motivation}

As mentioned frequently, monocrystalline (c-Si) based solar cells contribute to about $80 \%$ of the worldwide production volume of photovoltaic (PV) cells ${ }^{1}$. Silicon Nitride ( $\mathrm{SiN}$ ) is mostly used as antireflection (AR) coating for the front side and also as passivation coating for both front side

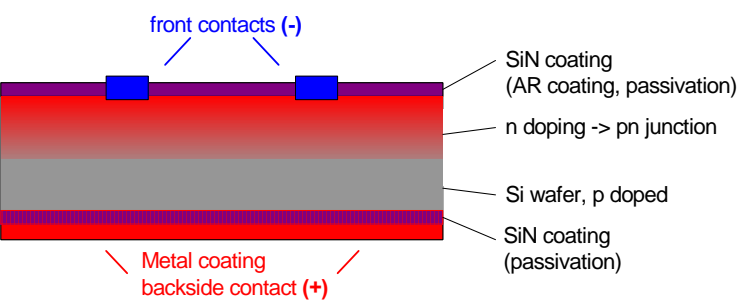

Fig.1 Classical Silicon PV cell structure. SiN acts as passivation and antireflection coating and must be reopened for electrical contacts

and backside. Because coating is done in large-area coating step (typical PECVD) and it is non-conducting, selective reopening of this layer is required as a preparation for the subsequent electrical contacting by screen-printing or metallisation coating ${ }^{2,3}$. A principal schema is shown in fig.1.

Laser ablation has some advantages for thin film removal: It is an ablation method free of mechanical contact, and thus free of tool wear-out. It is selective and introduces energy to the wafer only at the point of interaction. It is high-speed, because the mass of the motion system can be minimized (for a typical PV wafer size, the motion system is only a galvoscanner for beam deflection with tiny lightweight deflection mirrors). And it easily allows high-speed control and reproducibility of laser parameters which supports process automation.
One major demand of thin film processing is to prevent lattice damage, e.g. caused by melting. Melting effects are generated by heat conduction in the silicon. They can be minimized by using ultra short laser pulses with picosecond (ps) or femtosecond (fs) pulse duration. In that case absorption becomes non-linear ${ }^{4}$. This means that there occurs a significant component of the absorption coefficient $\alpha$ with the square of the peak intensity. This is efficient for ultra short pulse lasers, if the intensity exceeds about $10^{10} \mathrm{~W} / \mathrm{cm}^{2}$.

$$
\alpha=\alpha_{1} I_{0}^{1}+\alpha_{2} I_{0}{ }^{2}
$$

In the PV industry now UV ps laser sources are introduced. Because of lifetime effects in the UV converting crystal and the beam delivery optics, the use of NIR laser

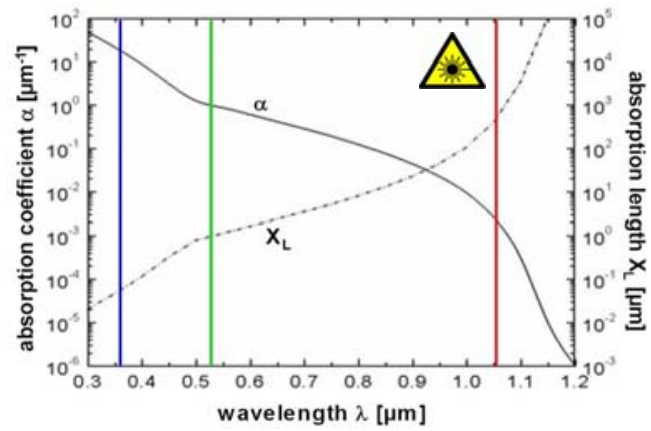

Fig.2 Optical absorption spectra for various VIS/NIR wavelength. Absorption length for $1025 \mathrm{~nm}$ wavelength is about $200 \mu \mathrm{m}$

sources in the range 1020 - $1070 \mathrm{~nm}$ should have advantages. Absorption for NIR wavelength is lower than for UV (fig.2), but shorter pulse duration of fs pulses result in higher nonlinear absorption. Thus we have investigated effect of fs ablation. 


\section{Thin film ablation with fs laser source}

Nonlinear absorption process is typical for pulse duration of less than $10 \mathrm{ps}$. In this timeframe the classical (thermal) ablation mechanism warming-up/melting/eva-poration skips to another effect: Some free electrons in the sample act as absorption centres and rapidly excite further electrons by Avalanche cascade process. Hot electrons dissipate into the environment of the excitation spot and thermalize by frequent collisions with other electrons. During that period there is a hot electron temperature and a cold lattice temperature. Finally the electrons transfer energy to the lattice within 0.5-5 ps and start the lattice heat conduction (which is caused by coupling of lattice atom vibrations). Because it is a lattice effect, melting will not occur until energy is transferred.

This means, if the pulse duration is shorter than the transfer time, then within a time period smaller than $10 \mathrm{ps}$ hot unbound electrons are released, which causes reduction of charge shielding of the lattice atoms. If the electrostatic repulsion force is too large, the lattice disintegrates locally by „Coulomb explosion“4 ${ }^{\star 4}$ ablation without melting. Effective heat conduction by free electrons can be neglected, because travel range within $10 \mathrm{ps}$ is in the order of $10 \mu \mathrm{m}$.

This principle works with all materials, independent of conducting, semi conducting or dielectric behaviour. Differences in the behaviour are mainly given by the specific electron-lattice energy transfer times.

Thus we have investigated SiN layer ablation by NIR femtosecond pulses. For this we used a commercial laser (JenLas D2.fs by JENOPTIK), which generates $40 \mu \mathrm{J}$ pulse energy within 400 fs laser pulses. Productivity demands can be matched with $100 \mathrm{kHz}$ repetition rate. A principal schema is given in fig.3.

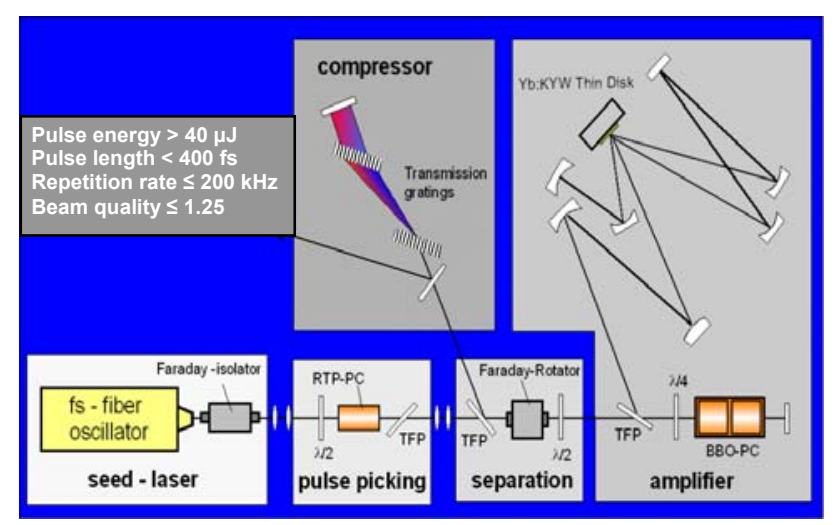

Fig.3 D2.fs laser by JENOPTIK uses a regenerative amplifier schema. $40 \mu \mathrm{J}$ pulse energy can be generated at $100 \mathrm{kHz}$ repetition rate.

To prevent melting effects caused by a cw-offset of the laser (between the pulses), careful layout and timing control of the oscillator-amplifier combination is required. Because there is no detector, which can measure fs pulse shape directly, autocorrelation measurement is state-of-theart. The trace is depicted in fig.4. We have not measured cw offset of the laser, but it is obvious from fig. 8 that it is not relevant for this application (no ablation trace between pulse ablation).

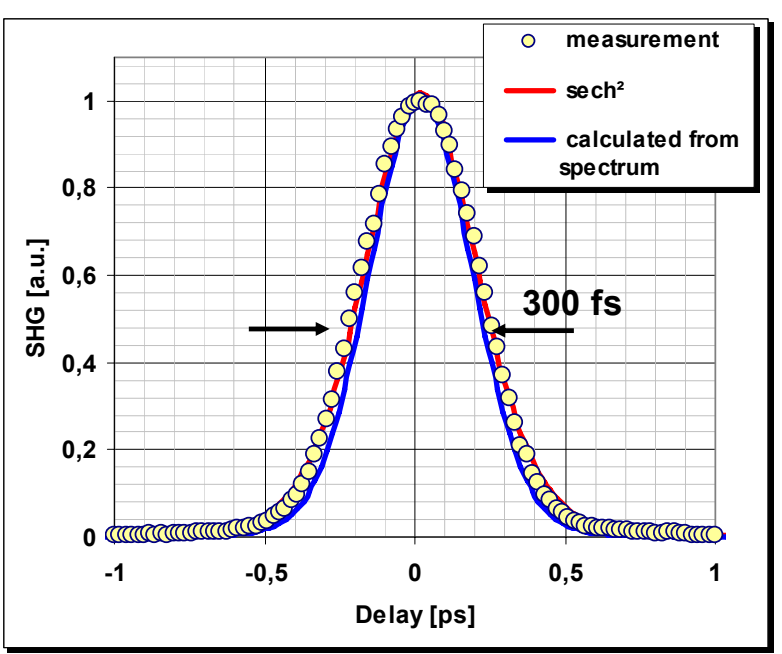

Fig.4 Pulse trace of D2.fs laser shows excellent 300 fs autocorrelation trace without $\mathrm{cw}$ offset.

\section{Application results}

Our optical beam delivery is described in another paper of LAMP 2009 proceedings ${ }^{5}$. Using $254 \mathrm{~mm}$ focal length plane-field optics by JENOPTIK, the working field is $160 \times 160 \mathrm{~mm}^{2}$, which is large enough for the full-size processing of a standard PV cell wafer $\left(156 \times 156 \mathrm{~mm}^{2}\right)$. Beam profile is Gaussian with $\mathrm{M}^{2} \sim 1.2$.

In fig.5 we show an application result: The ablation spot of a single laser pulse shows excellent ablation behaviour without visible melting effects.
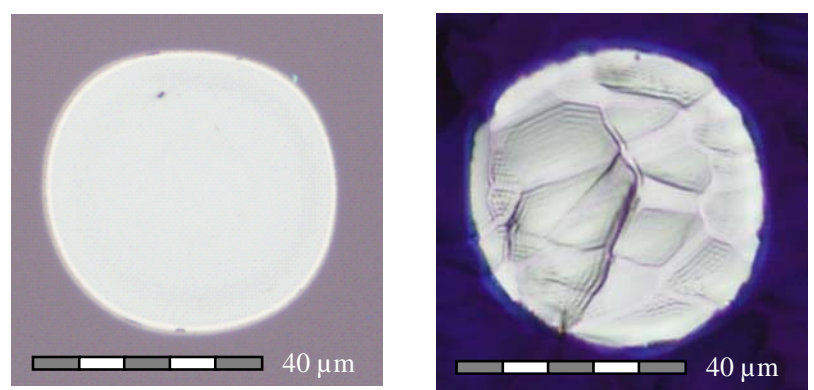

Fig.5 Application of $400 \mathrm{fs}$ pulses on different substrates without post-processing. Single-shot ablation without melting effects at c-Si (left) and m-Si silicon substrates (right). At multicrystalline substrates the grain boundary is not influenced by laser ablation.

Also a very sharp boundary of the ablation spot should be noted. In fig.6 a diagram is given describing the ablation diameter depending on the laser fluence.

The maximal applicable laser fluence is $0.35 \mathrm{~J} / \mathrm{cm}^{2}$; it is limited by melting effects which are visible by optical microscope (500x total magnification). More detailed analysis beyond optical microscopy, such as SEM and RBS, are under way and will be presented in a later publication.

Melting can be prevented, if the laser fluence undershoots a critical limit (fig. 6). 


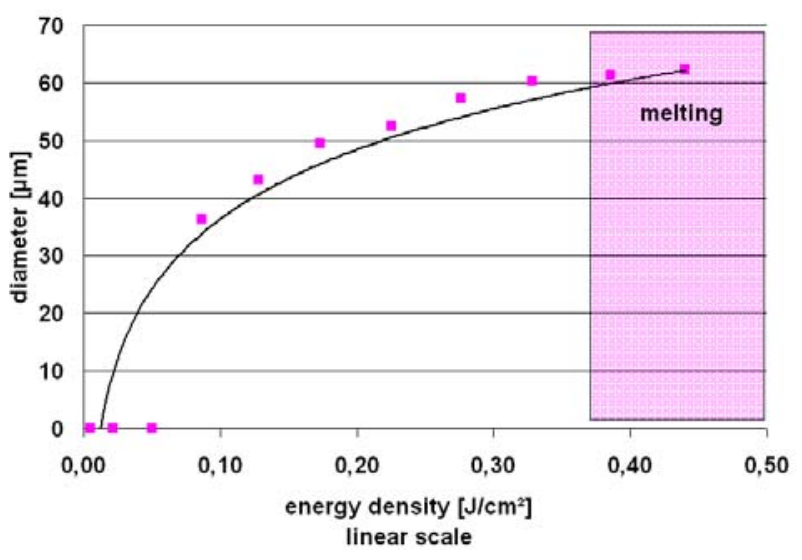

Fig.6 Diameter of ablation spot for 50-60 nm SiN coated to c-Si substrate. Maximum diameter is $60 \mu \mathrm{m}$; melting limit is $0.35 \mathrm{~J} / \mathrm{cm}^{2}$.

Since it is not easy to investigate melting of SiN on polished c-Si, we have also investigated the ablation of SiN, which was coated to a $\mathrm{KOH}$-etched Si surface. This etching step generates pyramidal surface structures - used as standard method to enhance the absorption of the PV cell. Because of the alternating orientations of the sample surface there is an enlarged efficiency of the PV cell due to the changing incidence angle of the sunlight to the cell surface. In fig.7 it can be seen, that inside the circular ablation spots there is complete ablation of the SiN layer and excellent sharp ablation boundary. Conservation of the microstructure is a proof of melting-free ablation.

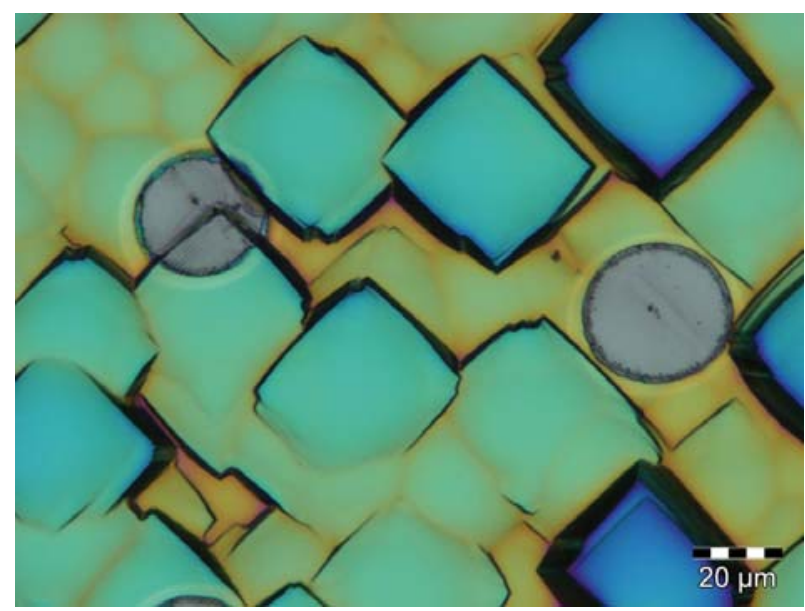

Fig.7 KOH etching of c-Si shows pyramidal surface structures generated by silicon lattice planes. Single-shot femtosecond laser processing (round spots) shows conservation of these structures without melting.

Not only the laser fluence limit has to be taken into account for prevent melting, but also the pulse length. Thus we have compared in a first principal trial the performance of a 400 fs laser with a 10 ps laser at comparable wavelength (1025 and $1064 \mathrm{~nm}$ ). We held the laser fluence constant for the result shown in fig.8. It is clearly visible, that the ablation with ps NIR laser shows melting effects and a bad definition of the ablation spot boundary. We assume that electron-lattice energy transfer time is significantly less than 10 ps and thus there is not enough

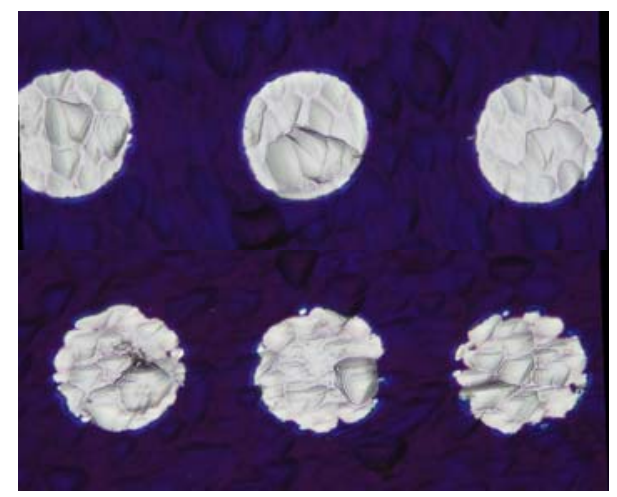

Fig.8 Comparison of a 400 fs (above) and 10 ps (below) ablation using NIR lasers. The ps laser shows melting effects and bad definition of ablation boundary

nonlinear absorption in the $\mathrm{SiN}$ coating. Because $\mathrm{SiN}$ is designed as AR coating, for low intensities it becomes transparent for NIR. This way a share of the pulse energy is transmitted through the SiN coating and finally absorbed in the Silicon bulk material below, causing a lattice temperature enhancement and finally melting which can be observed.

Because nonlinear absorption data for the SiN/Si layer system are not known, a systematic investigation is required. Our intention for further trials is to set up a laser which allows tuning of pulse duration between $600 \mathrm{fs}$ and 10 ps.

\section{Summary}

We have demonstrated "cold" ablation of $50-80 \mathrm{~nm}$ thick SiN layers by use of a NIR femtosecond laser (JenLas D2.fs by JENOPTIK). Single-shot principal trials show damage-free ablation of circular spots with up to $60 \mu \mathrm{m}$ diameter. This ablation can be performed with $400 \mathrm{fs}$ pulses up to $100 \mathrm{kHz}$ repetition rate. Use of NIR-picosecond laser shows significantly worse ablation quality.

More detailed analysis of above results of ablation properties of the SiN/Si system are under way and will be presented later as well as systematic tuning of the ultra short pulse duration to the ablation quality.

\section{References}

[1] ${ }^{1}$ B.Sopori: Silicon nitride processing for control of optical and electronic properties of silicon solar cells, Journal of Electronic Materials, Vol. 32, Nr. 10, 2003, pp. 1034-42

[2] ${ }^{2}$ F.Book et al.: Two Diffusion Step Selective Emitter: Comparison of Mask opening by Laser or Etching Paste, 23rd EC PVSEC, September 1-5, 2008, Valencia

[3] ${ }^{3}$ K.Neckermann et al.: Local Structuring of Dielectric Layers on Silicon for improved Solar Cell Metallisation, $22^{\text {nd }}$ EPSEC, September 3-7, 2007, Milano

[4] ${ }^{4}$ F.Dausinger et al., Femtosecond Technology for Technical and Medical Applications (Springer, Berlin/Heidelberg, 2004), pp.105

[5] ${ }^{5}$ K.Stolberg et al., Systematic optimisation of process parameters in laser drilling..., JLMN- Journal of Laser Micro/Nanoengineering, Vol.4, Nr.3, 2009, pp. 231-233

(Received: July 10, 2009, Accepted: March 16, 2010) 